Article

\title{
A Survey on Integration of Optimization and Project Management Tools for Sustainable Construction Scheduling
}

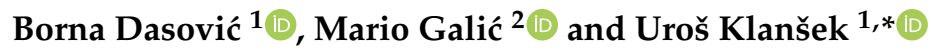 \\ 1 Faculty of Civil Engineering, Transportation Engineering and Architecture, University of Maribor, \\ Smetanova ulica 17, 2000 Maribor, Slovenia; borna.dasovic@um.si \\ 2 Faculty of Civil Engineering and Architecture Osijek, Josip Juraj Strossmayer University of Osijek, Ulica \\ Vladimira Preloga 3, 31000 Osijek, Croatia; mgalic@gfos.hr \\ * Correspondence: uros.klansek@um.si; Tel.: +386-2-22-94-378
}

Received: 3 April 2020; Accepted: 16 April 2020; Published: 22 April 2020

\begin{abstract}
Construction scheduling, in practice, commonly relies on the usage of commercial project management tools (PMT) without specific optimization features. To obtain optimal schedules, planners often need to develop separate optimization models with special tools, which, however, demand further processing and editing of optimization results by PMT into forms expected for project management. In this regard, separation of optimization and PMT also requires considerable additional work for complete and harmonized updating of schedules during construction execution. Mentioned drawbacks and lack of available time may take to deficient construction scheduling during the implementation phase resulting in poor or even insufficient realization of project goals. Therefore, this paper presents an achievements survey on the integration of optimization and PMT that allow sustainable construction scheduling, particularly in terms of continuous optimal time and resource allocation throughout the project life cycle. Such work has not yet been comprehensively done up to now and the present contribution intends to fill a literature gap in the aforesaid area. Following a brief introduction, the optimization platform for construction scheduling is given in the article. Focusing on construction scheduling, an in-depth achievements survey on the integration of heuristics methods, mathematical programming and special solving methods with conventional PMT as well as optimization-based building information modeling (BIM) tools is then performed and findings are reported. The paper ends with conclusions and recommendations for further research.
\end{abstract}

Keywords: sustainable scheduling; construction execution; optimization; project management tools; software integration; building information modeling; BIM

\section{Introduction}

Sustainable construction can be recognized as the practice of creating facilities and applying procedures that are environmentally sound and resource-efficient throughout the life cycle of a building from siting to design, construction, operation, maintenance, renovation and deconstruction [1]. In order to ensure sustainable operation management during the construction phase, the main objective of project managers is to create sustainable schedules. A sustainable schedule must continue to meet all deadlines, even if parameters change during the task's execution period [2]. In other words, sustainable scheduling is not only about delivering a result according to expectations, within the available budget and on time, but also about ensuring that decisions are sustainable in the long term [3].

Sustainable scheduling, in terms of continuous optimal time and resource allocation throughout a project's life cycle, is broadly recognized as a relevant topic of construction management. Namely, 
construction processes are usually labor-intensive and frequently require the large-scale engagement of costly resources. Construction is often characterized by a diversity of contracted works that demand various participants, many of whom are also simultaneously burdened by activities in their other dealings. In addition to that, influenced by location-specific conditions and weathering, construction projects are commonly carried out in stochastic and dynamic environments. All said particularities, and the fact that wrong decisions taken in high-value construction can seriously compromise business success or even contractor's existence on the market, clarify exposed need of the building industry to engage optimization-supported project management tools (PMT) into scheduling tasks.

The building site is a changing environment and it is expected that the originally created schedule will undergo many modifications during the construction process. In addition, construction schedules often contain a large number of activities. This way, resource utilization options, at the activity level, generate an exponentially increasing number of possible schedule combinations in regard to the growth of the number of construction activities. Besides being mathematical, this tends to be a practical issue as well.

Due to their dynamic environment, projects in the construction industry pose a challenge that is reflected in the time-consuming process of planning and updating, which is usually carried out on a daily or weekly basis. This problem becomes most obvious when a project reaches the construction phase, where the number and frequency of design changes rise $[4,5]$, whilst directly affecting the schedule by demanding its revisions and establishing new activities and relations. Therefore, the motives for achieving a higher level of automation in construction scheduling (e.g., through the integration of optimization and PMT as addressed here) are clear and can be summarized as a simple goal: to gain quantitatively assessed information (i.e., schedule) as quickly as possible regardless of project complexity or phase.

In conventional construction practice, commercial PMT without specific optimization features are commonly employed for scheduling of contract works. In order to achieve effective construction schedules, planners thus usually perform rather time-consuming iterative procedures within PMT providing a single or even a set of feasible (i.e., sub-optimal) solutions, or they develop separate optimization models. Challenging as they are, optimization models for scheduling problems have been intensively developing for decades and reviews of the state of the art on this field may be found in published sources [6,7]. Time-cost tradeoff problems [8], resource-constrained scheduling problems [9], resource leveling problems [10], scheduling problems with time-cost-risk tradeoffs [11] as well as their extended or modified variants can be identified as those that have been most frequently taken into consideration.

However, the difficulty of data collection and processing has been significantly less addressed as an issue of developing separate optimization models for scheduling. Data input in such models tend to require significant amount of time and labor. Thus, a method to transfer data from the data source to the problem's mathematical model is necessary, as pointed by the authors [12]. Another shortcoming of the aforementioned models is that the optimization results demand further processing and editing by PMT, providing forms and formats anticipated in the project management. In this regard, separation of optimization and PMT also requires considerable additional work for complete and harmonized updating of schedules during construction execution.

Mentioned drawbacks and lack of available time, due to the dynamic nature of input parameters and frequency of necessary updates, may take to deficient construction scheduling during the construction phase resulting in poor, or even insufficient realization of the main project goals. Therefore, this paper presents an achievements survey on the integration of optimization and PMT that allow sustainable construction scheduling, particularly in terms of continuous optimal time and resource allocation throughout the project life cycle. Furthermore, the survey aims to demonstrate current developments in the process automation of scheduling, plan analysis and updating whilst streaming into a form of active building information modeling (active BIM). As far as the authors' knowledge, 
such work has not yet been comprehensively done up to now whilst the main contribution intends to fill a literature gap in the aforesaid area.

In this way, the remainder of this paper is organized as follows. The list of abbreviations is introduced at the end of Section 1. Section 2 gives the optimization platform for construction scheduling (Table 1). Achievements on the integration of heuristics methods and PMT are presented in Section 3, which is thereupon followed in Section 4 by revealing developments on the integration of mathematical programming and PMT. Section 5 is particularly devoted to the integration of special solving methods and PMT, and Section 6 addresses contributions on optimization-based BIM tools. Supported by survey findings, the paper ends with Section 7 , which draws conclusions and recommendations for further research.

Table 1. Abbreviations.

\begin{tabular}{|c|c|}
\hline $\mathrm{ACO}$ & Ant Colony Optimization \\
\hline ACS & Ant Colony System \\
\hline API & Application Programming Interface \\
\hline ASS & Advance Scatter Search \\
\hline BB & Branch-and-Bound \\
\hline $\mathrm{BC}$ & Branch-and-Cut \\
\hline $\mathrm{CP}$ & Constraint Programming \\
\hline $\mathrm{CH}$ & Constructive Heuristics \\
\hline ESS & Early Start Schedule \\
\hline FBHA & Forward-Backward Heuristic Algorithm \\
\hline GA & Genetic Algorithm \\
\hline GRASP-HH & Greedy Randomized Adaptive Search Procedure Based Hyper-heuristics \\
\hline HS & Harmony Search \\
\hline IFC & International Foundation Classes \\
\hline JITPS & Just-In-Time Production System \\
\hline JSP & Job-Shop Problem \\
\hline LP & Linear Programming \\
\hline LSS & Late Start Schedule \\
\hline MCS & Monte Carlo Simulation \\
\hline MILP & Mixed Integer Linear Programming \\
\hline MOSP & Multi-Objective Scheduling Problem \\
\hline MRCPSP-PCF & Multi-mode Resource-Constrained Project Scheduling Problem with Positive Cash Flows \\
\hline MRDH & Multi-start Random Descent Hyper-heuristics \\
\hline PF & Pareto Front \\
\hline PSO & Particle Swarm Optimization \\
\hline PSS & Project Selection Strategy \\
\hline RACP & Resource Availability Cost Problem \\
\hline RAP & Resource Allocation Problem \\
\hline RAPSP & Risk-Aware Project Scheduling Problem \\
\hline RCPSP & Resource-Constrained Project Scheduling Problem \\
\hline RLP & Resource Leveling Problem \\
\hline SA & Simulated Annealing \\
\hline SDESA & Simplified Discrete Event Simulation Approach \\
\hline TA & Threshold Accepting \\
\hline TCTP & Time-Cost Tradeoff Problem \\
\hline TS & Tabu Search \\
\hline VBA & Visual Basic for Applications \\
\hline
\end{tabular}




\section{Optimization Platform for Construction Scheduling}

Optimization, as a wide range of exact mathematical programming, heuristics, meta- and hyper-heuristics methods, is proven capable of sustainable quantitative decision-making process providing solutions in various fields including construction scheduling. Notwithstanding that optimization problems may differ from one another, they can all be generally formulated with an objective function to minimize $f(x)$, subjected to $h(x)=0$ and $g(x) \leq 0$, where $f(x)$ represents the objective function of decision variables $x$ while $\boldsymbol{h}(x)=\mathbf{0}$ and $g(x) \leq \mathbf{0}$ indicate equality and inequality constraints. In this context, the objective function expresses the criterion by which the optimal solution is selected while the constraints determine boundaries for the region of all feasible solutions. The objective function can also be maximized over the feasible region if that fits better with the nature of the optimization criterion. As far as decision variables are concerned, their values should be found among the placed lower and upper bounds, $x^{\mathrm{LO}} \leq x \leq x^{\mathrm{UP}}$, and they can be either declared as continuous or as integer. Optimal discrete decisions can be taken applying binary $0-1$ variables.

In general, most scheduling optimization problems nowadays are considered as NP-hard, and as such they are challenging in terms of choice and utilization of solving algorithms. From viewpoint of construction scheduling optimization problem, input data to be generated into its model may comprise sets of project activities and precedence relations among them as well as time, cost, resource and other options related to possible execution modes. Decision variables, for which the optimal values should be identified by schedule optimization, often represent start times and durations of activities, lag and lead times, project duration, quantities of resources, etc. Binary variables are usually included in the model for selecting scenarios of optimal production processes, execution modes of activities, working time units and other discrete choices that make an impact on the efficiency of construction schedules. Optimization of aforesaid variables can be carried out under various relevant objectives, although the minimization of total cost in regard to project duration seems to be among the most commonly chosen criteria in practice [13].

The total cost objective function of the construction scheduling problem is often formulated to include direct costs of employed resources (e.g., labor, machinery, equipment, etc.) as well as indirect costs (e.g., business overheads, operating costs, etc.). In addition to said costs, penalties and bonuses, if they are agreed by the construction contract, may significantly influence the cost-optimal scheduling too. Then, in fact, the penalty cost may be incurred if the construction is delayed after the contracted deadline, while the bonus can be earned if the construction is completed earlier than agreed. All of that consequently requires the contract penalties and bonuses to be also covered within the total cost objective function.

In any case, the optimal solution of the construction scheduling problem needs to satisfy equality and inequality constraints as well as bounds on decision variables determined in the optimization model. At this point, the constraints can be defined for generalized precedence relations, activity durations and start times, lag and lead times, execution modes, project duration, assignments of working time units, engagement of resources, etc. As regards to bounds on the aforementioned scheduling variables, these are typically needed to set upper and lower limits on their values, thus closing the region of feasible solutions. Optimization models developed for construction scheduling are expected to provide output data expressed with (near) optimal values of decision variables useable for making normal graphical representations relevant to project management, such as network diagrams, Gantt charts, histograms, S-curves and others [14].

A wide range of commercially available software exists for computer modeling of construction scheduling optimization problems in forms of algebraic modeling languages, spreadsheets, interactive computer languages and applications. A comprehensive review of contemporary modeling tools and optimization algorithms supported with a discussion about their applicability, advantages and limitations can be found in references $[15,16]$. 
With a particular focus on construction scheduling, the following sections report findings of an in-depth survey devoted to the integration of heuristics methods, mathematical programming and special solving methods with conventional PMT as well as optimization-based BIM tools (Figure 1).

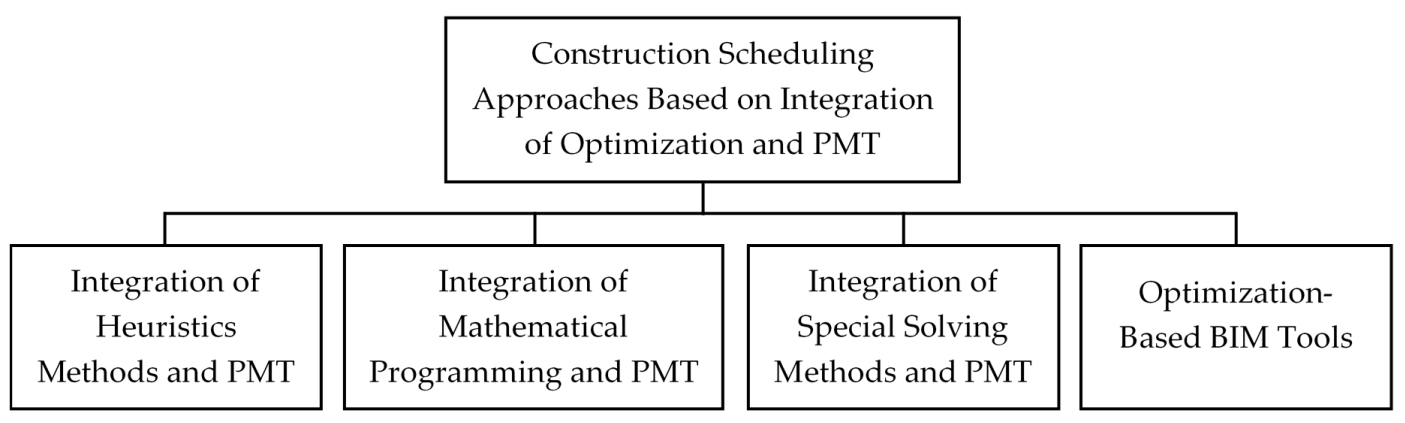

Figure 1. Construction Scheduling Approaches Based on Integration of Optimization and PMT.

Levels of said integration used in below sections are classified as follows:

- Moderate: input data from PMT and/or other software are imported into an optimization model, while the optimization results have to be edited manually in PMT to ensure their display.

- Medium: input data from PMT and/or other software are imported into an optimization model, while the optimization results are then either automatically transferred or exported back to PMT, ensuring their display with minor data adjustments.

- High: generation of input data, optimization process and display of output results are fully performed within PMT.

\section{Integration of Heuristics Methods and PMT}

In general, most heuristics methods have shown the capacity to solve a diverse range of scheduling tasks. Despite their wide usability, heuristics algorithms often report approximate (near) optimal solutions after the search process is completed whilst strengthening the importance of the post-optimization analysis process. Their major advantages are that most of them demonstrate reasonably fast convergence and can cope with optimization problems that include non-differentiable expressions. However, due to the complexity of scheduling problems and reported drawbacks of heuristics algorithms regarding their problem-dependent efficiency, meta-heuristic algorithms have shown to be more promising. Achievements on the integration of heuristics methods (i.e., heuristic, meta- and hyper-heuristic) and PMT are summarized in Table 2.

Integration of heuristics optimization techniques and PMT attracted considerable attention among researchers. Early studies of Hegazy [17,18] addressed the implementation of the GA in the macro language of commercial PMT, the early version 4.1 of Microsoft Project. The said research was devoted to solving TCTPs [17], RAPs and RLPs [18], i.e., optimization tasks which often encounter in construction scheduling. It was concluded that implementation of the GA with existing PMT brings direct benefits for the development process and users. The main downside that the author pointed to was the randomness nature of the algorithm, which required a long processing time for large networks. The use of faster programming languages, such as $\mathrm{C}$ and $\mathrm{C}++$, was suggested as a direction for further research.

Leu et al. [19] developed a GA-based system for solving RLPs, called GARLS. The system was consisted of three components: database, user interface and model base. These components operate together to process the construction scheduling and the balancing of production resources. The results revealed that the suggested approach ensures a decision support system adequate for industrial application. Limitation of this study lies in the general and well-known imperfection of heuristics, i.e., their potential risk of providing a feasible or near-optimal solutions. 
Crawford et al. [20] created a software package, SWORD, intended to the optimal project selection for publicly funded research. The software incorporates many aspects in the process of project selection, i.e., cost, probability, utility and technical constraints between projects. The authors combine MCS and an optimization component that uses ASS and TS. First, simulations show the likelihood of the specific outcome, then optimization is employed to search a small subset of actual solution space. The conclusion is that the presented software can serve as a decision support tool also in industrial environments. It has to be stated that the authors have not used this software on a real-life problem but only investigated the possibilities of its application.

Table 2. Research on Integration of Heuristics Methods and PMT.

\begin{tabular}{|c|c|c|c|c|c|}
\hline Year & Ref. & Scheduling Problem & Optimization Approach & Project Management Tool & Level of Integration \\
\hline 1999 & [17] & TCTP & GA & MS Project & High \\
\hline 1999 & [18] & RAP and RLP & GA & MS Project & High \\
\hline 2000 & [19] & RLP & GA & MS Project & Medium \\
\hline 2003 & [20] & PSS & MCS, ASS and TS & MS Project & Moderate \\
\hline 2003 & [21] & MOSP & GA & MS Project & High \\
\hline 2003 & [22] & RAP & GA and simulation & MS Project & High \\
\hline 2005 & [23] & Multi-objective TCTP & GA & MS Project & High \\
\hline 2006 & [24] & MOSP & GA & MS Project & High \\
\hline 2006 & [25] & TCTP & GA & MS Project & Medium \\
\hline 2007 & [26] & TCTP & ACS & MS Project & High \\
\hline 2008 & [27] & RCPSP & GA & MS Project & High \\
\hline 2008 & [28] & RLP & SDESA and PSO & $\begin{array}{c}\text { Simplified Simulation-based } \\
\text { Scheduling (S3) }\end{array}$ & High \\
\hline 2009 & [29] & JSP and RCPSP & GA and simulation & MS Project & High \\
\hline 2009 & [30] & RCPSP & GA & Primavera P5 & High \\
\hline 2010 & [31] & RLP & SA & MS Project & High \\
\hline 2010 & [32] & RCPSP & GA & MS Project & High \\
\hline 2012 & [33] & RLP and RAP & $\mathrm{SA}, \mathrm{TA}$, and MRDH & MS Project & High \\
\hline 2012 & [34] & ТСТР & ACS & MS Project & Medium \\
\hline 2012 & [35] & RCPSP & GRASP-HH, ACS, GA & MS Project & High \\
\hline 2013 & [36] & RCPSP & ESS, LSS and CH & MS Project & High \\
\hline 2013 & [37] & Multi-objective TCTP & HS & MS Project & Medium \\
\hline 2013 & [38] & JSP & TS & LibrePlan & High \\
\hline 2013 & [39] & RLP and RACP & TS & MS Project & High \\
\hline 2014 & [40] & RCPSP & GA & MS Project & Moderate \\
\hline 2014 & [41] & RCPSP & ESS, LSS and $\mathrm{CH}$ & MS Project & High \\
\hline 2015 & [42] & RCPSP & GA & MS Project & Moderate \\
\hline 2015 & [43] & Multi-objective RCPSP & GA & MS Project & Moderate \\
\hline 2018 & [44] & MOSP & GA & MS Project & High \\
\hline
\end{tabular}

Dawood and Sriprasert [21,24] introduced a multi-constrained scheduling system that takes into account construction conditions, i.e., physical, contractual, resource and information constraints. Additionally, the objective function was constructed of four separate objectives for duration, cost, resource and space utilization, each represented with provided weight factor. The authors have developed the software application using the VBA language in MS Project and demonstrated the advantages of their approach on a practical example. Such software may assist the project planners in making their schedules more reliable in accordance with the constraints. Authors considered further research development in terms of implementing additional constraints, to investigate various formulation techniques to solve MOSP and improve the GA mechanisms to solve more complex problems. 
In their paper, Hegazy and Kassab [22] combined GAs with process-flow simulation in order to improve resource usage and construction planning. Three different software were used: MS Project as a PMT, Process V3, a process simulation software, and a commercial GA-based optimization tool called Palisade Evolver. The software was incorporated using two VBA modules into MS Project. The proposed approach was tested on two examples: the placement of concrete columns and the use of earthmoving equipment in Hong Kong International Airport. It was pointed out that the progressiveness of this approach lies in its automated performance and simplicity.

To solve multi-objective TCTP, Zheng et al. [23] introduced innovative techniques into the GA model, i.e., PF ranking, niche formation and adaptive mutation rate. Their modified adaptive weighting approach, known as MAWA, overcomes the standard problem of integrating time and cost into a single objective. The suggested MAWA model was developed using the macro language of MS Project. The authors have recommended additional objectives in future studies, such as quality and safety.

As published in their article, Kandil and El-Rayes [25] developed a multi-objective automated construction resource optimization system (MACROS). The system was created in Microsoft Visual $\mathrm{C}++$ to implement data from MS Project and MS Access. The GA was implemented in MACROS as a module to solve time-cost-quality tradeoff problems (enhanced TCTP). The capabilities of the system were demonstrated on the example of a highway construction project. The MACROS allow project managers to evaluate various combinations of weighting factors in terms of time, cost and quality; and it assists in determining which plan is to be implemented.

Zhang et al. [26] investigated the possibility of applying ACS in order to solve discrete TCTP of construction scheduling. The model was developed by VBA programming and linked to MS Project. The ACS algorithm was used to solve the problem discussed and was compared with some other evolutionary-based algorithms. However, it should be mentioned here that all the results were not gained on the same computer, which reduces possibilities for taking general conclusions.

The study by Lancaster and Cheng [27] dealt with the project scheduling problem that utilizes preferential logic optimization in order to meet resource requirements applied on a case study project of hydrotesting of newly constructed tank farms. In order to minimize the utilization of water, the author's original fitness differential adaptive GA was used. The authors translated the algorithm from the original MATLAB code into the VBA programming language and implemented it in MS Project. Applied on a practical example, the proposed approach yielded a feasible solution and leveled the resources. The further development of a model to solve MOSP was suggested.

Lu et al. [28] developed a Simplified Simulation-based Scheduling system (S3) to solve RLP. The presented software combines SDESA and PSO algorithm to automate the formation of a resource-constrained schedule with the shortest overall project duration. The authors demonstrated the advantages of their solution in relation with commercial PMT. The software was tested on a textbook example and on an actual drainage project in Hong Kong. Both examples showed significant improvements in overall project duration and resource use. It was mentioned that the further system development will be directed to TCTPs and the use of PF, MCS, as well as a multi-objective analysis.

Zülch et al. [29] compared GA solutions of JSP and RCPSP with the results of the planning procedure REIMOS (German abbreviation for "Sequence planning for multi-product manufacturing systems") and the simulation with FEMOS (German acronym for "Manufacturing and assembly simulator"). The tools were integrated into the MS Project as add-ins, in order to make it more user-friendly. One of the possibilities that emerged from this study was to combine simulation and optimization methods in such a way that the simulation tool could be used as a fitness function of the GA.

While most studies used MS Project for project management, Kim and Elis [30] have examined another long-established PMT, i.e., Oracle Primavera P5. The authors have created an add-in function within Primavera that runs the elitist GA to solve the RCPSP. The functionality was demonstrated on a coke bunker construction project. The proposed model has shown some efficiency limitations, 
e.g., it was capable to provide optimal results for up to 60 activities and near-optimal results for a project with up to 120 activities.

Anagnostopoulos and Koulinas [31-33,35,39] have presented an in-depth analysis with their series of papers. The authors have dealt with various scheduling problems and solved them with several heuristic algorithms, i.e., SA, GA, TA, MRDH, GRASP-HH, ACS and TS. All models were developed within MS Project while optimization algorithms were created in VBA. This series of studies have shown the relevance of research in this area. The improvements were aimed at faster performance through more efficient learning mechanisms and more sophisticated low-level heuristics.

Another example of an ACS-based model for the TCTP of construction scheduling was discussed by Zhang and $\mathrm{Ng}$ [34]. Authors deployed previously described MAWA to optimize both time and cost simultaneously. The model was coded in VBA and plugged to MS Project. MS Excel was used for result analysis. The model was described as user-friendly and efficient, making it easy for project managers to apply it to specific construction projects.

Guedes and Tereso [36,41] used the programming language C Sharp (C\#) to develop MS Project add-in function with various algorithms to solve RCPSP. The algorithms discussed were ESS, LSS and $\mathrm{CH}$. Additionally, the MS Project option for resource-leveling was considered. ESS and LSS algorithms already exists within the MS Project and they are capable to arrange all activities as soon as possible or as late as possible, respectively. The $\mathrm{CH}$ procedure consists of three basic parameters, i.e., a priority rule, a scheduling scheme and a schedule direction. It was noted that the $\mathrm{CH}$ method obtains different results using different parameters, hence all possible combinations should be considered solutions' assessment in order to define which one provides the best result. The proposed approach demonstrated an advantage in terms of selecting between several algorithm alternatives.

In their study, Moselhi and Roofigari-Esfahan [37] used MATLAB with a dynamic link to MS Project for the purpose of solving the multi-objective TCTP. The authors employed the HS method that tends to find the best set of project activity alternatives through the generation of harmony memory matrix and updating this matrix by replacing each activity's alternative with one that better satisfies the objective of gaining less total project cost. The limitation of the proposed approach found was that it can only be applied to linear projects with a series of repetitive actions.

The paper of González-Sieira et al. [38] demonstrated an innovative implementation of the scheduling module within the web-based open-source PMT called LibrePlan. TS algorithm was formed in Java programming language to optimally solve JSP. However, it was noted that this methodology likely requires advanced programming skills, which limits the number of project managers who can use it on construction projects.

Karova et al. [40,42] created an application called OPTPROJECT that uses MS Project file as an asset to generate input data for the optimization model. The program serves as a graphical interface for project management and also as an optimization tool. Execution of the GA optimization results with output information in suitable graphical form. It was found that further implementation steps for a higher degree of automation should be addressed in future work.

Kaiafa and Chassiakos [43] established a complex model for solving multi-objective RCPSP to optimality. The model was developed in MS Excel and utilizes GA programmed in VBA. The objective of the optimization was minimization of the overall costs generated by additional expenses, i.e., resource overallocation and day-to-day fluctuations, as well as project deadline exceedance. The optimization results have been presented in MS Project illustrating the Gantt chart and resource histogram chart. It was found that the solving efficiency of proposed method decreases as the number of activities or resource types increases but it was noted that even the slightest improvements in resource utilization can lead to a notable return.

In their study, Eid et al. [44] were focused on linear infrastructure projects with repetitive activities. The data input was done via the MS Project. The VBA macro module with GA and PF sort implementation-allowed schedule altering based on given inputs. The model was tested on the project of a three-span concrete bridge and on an example of highway construction. It took into account which 
crew should be deployed for minimum project cost and overall project duration while minimizing project disruption and delays. However, only finish-to-start precedence relationships between activities were examined and the model was found applicable only for linear projects.

\section{Integration of Mathematical Programming and PMT}

Mathematical programming (i.e., exact optimization algorithms) are used as advanced tools for optimal scheduling, as well. The main beneficial characteristic of mathematical programming methods is that they provide the exact optimal solution though the optimization process itself and may, in some instances, take a longer time to be concluded. Slower convergence of exact optimal solution can especially occur in dealing with highly combinatorial, discrete, nonlinear and nonconvex problems. Developments on the integration of mathematical programming and PMT are revealed in Table 3.

Table 3. Research on Integration of Mathematical Programming and PMT.

\begin{tabular}{llcccc}
\hline Year & Ref. & Scheduling Problem & Optimization Approach & Project Management Tool & Level of Integration \\
\hline 2011 & {$[45]$} & TCTP & Simplex & MS Project & Moderate \\
\hline 2013 & {$[36]$} & RCPSP & BB & MS Project & High \\
\hline 2014 & {$[41]$} & RCPSP & BB & MS Project & High \\
\hline 2015 & {$[46]$} & TCTP and RCPSP & BB & MS Project & Moderate \\
\hline 2016 & {$[47]$} & TCTP & BC & MS Excel ${ }^{1}$ & Medium \\
\hline 2017 & {$[48]$} & TCTP & Simplex & MS Project & Medium \\
\hline $\begin{array}{l}\text { Notes: } \\
\text { tracking projects. }\end{array}$
\end{tabular}

While the interest on the integration of mathematical programming and PMT seems not as widely presented, as it was in the case with heuristics, the following studies have investigated this possibility. In their research, Hebert and Deckro [45] combined MS Project with the traditional LP model to solve a TCTP. The model was developed in MS Excel and solved using Excel Solver add-in. The objective of the optimization model was to shorten the project duration by a certain number of days at a minimum cost. The model included generalized precedence relations between activities, i.e., finish-to-start (FS), start-to-start (SS) and finish-to-finish (FF). The functionality of this approach was shown on the example of a small-scale construction project. Capabilities of MS Project with the author's original LP model are combined to revise customer requirements for the shortened project duration. The results of the time-cost tradeoff analysis are then entered back to MS Project to obtain an updated construction plan. The authors note the anomaly that occurs in the specific case of FF and SS dependencies that requires extending activity duration to shorten the overall duration of a project. The main limitation of this study is manual data manipulation and a presumably highly complex spreadsheet-based optimization model. The minor changes in project activity dependencies or change in the number of activities would result in excessive model modifications that are hardly practical in day-to-day use in construction management.

Guedes and Tereso's $[36,41]$ approach was already mentioned in Section 3. The authors created an MS Project add-in function using a C\# programming language to implement heuristics methods but also an exact mathematical programming technique, i.e., BB algorithm. The results showed that there was no great variation between heuristics and mathematical programming method results due to the low complexity of the project network. It should be noted that the BB algorithm analyzed all possible solutions within a single run while heuristics methods were susceptible to parameter sensitivity. In other words, it was found that all possible combinations between parameter preferences should be investigated to obtain the best possible result. Further improvements were suggested in terms of user interface and other optimization approaches to the RCPSPs.

Tiwari and Johari [46] developed a MILP model to solve TCTP and RCPSP simultaneously. The model was established as a two-step procedure. First, MS Excel was employed to solve the TCTP without considering resource constraints and then MS Project was used to explore variations 
of schedules obtained in the first step to achieve the target overall project duration with available resources. This approach was demonstrated on a small-scale project. Information on project activities has been entered into MS Project. For the aim of minimizing the total project cost, Excel Solver add-in was enforced. While the employed Simplex LP solver is mainly intended for LP problems, it can be also used for MILP tasks and in that case, the solver engages BB algorithm. MS Project was used to show daily resource utilization. The main drawback of this study is that both software were employed separately so there was no automation in data transmission, and it raises the question about human work required to apply this approach in complex construction projects.

In a doctoral thesis, Siganporia [42] has considered optimization tools for production planning in biopharmaceutical facilities. The production process addressed was a complex task because every decision reflects on risks and production cost. This study describes a framework for a mathematical programming model that deals with capacity planning to minimize the total cost. The optimization model was developed in the General Algebraic Modeling System (GAMS) while the BC algorithm implemented through MILP solver CPLEX was used to solve the optimization problem. The input data was gathered in MS Excel with a two-way connection with GAMS. The GAMS can read the data from MS Excel and return the result to MS Excel. This integration was achieved via the GDXXRW tool. To show the Gantt chart of the optimization output schedule, code was written in the VBA programming language. It was noted that the drawback of MS Excel and GAMS implementation was editing. Any change in activity formulation or number of activities would lead to excessive and error-prone modifications in the mathematical programming model.

Valenko and Klanšek [43] created a spreadsheet-based LP model to solve TCTP. In this study, MS Excel was used for input data of project activities. The optimization model was also developed in MS Excel using the Excel Solver add-in to minimize the total project cost. The Simplex LP solver was applied to solve the LP problem engaging the simplex method. The proposed approach was tested on a textbook example and the optimal solution was obtained. The authors also created an application using VBA programming language to export optimal schedule to MS Project. That particular feature reduced possibilities of errors during project scheduling, though further development of the approach is necessary to achieve a higher level of integration between optimization and PMT.

\section{Integration of Special Solving Methods and PMT}

Alongside optimization methods, some special solving methods capable of providing high-quality solutions have been also developed and integrated with PMT to assure efficient construction scheduling. Published research works devoted to such integrations are listed in Table 4 giving the key information about software features applicable for construction scheduling.

Table 4. Research on Integration of Special Solving Methods and PMT.

\begin{tabular}{cccccc}
\hline Year & Ref & Scheduling Problem & Solving Method & Project Management Tool & Level of Integration \\
\hline 2013 & {$[49]$} & RLP & FBHA & MS Project & High \\
\hline 2014 & {$[50]$} & TCTP & C $^{2} S^{2}$ & Primavera P6 & Moderate \\
\hline 2014 & {$[51]$} & RAP & Simulation & MS Project & Moderate \\
\hline 2016 & {$[52]$} & JITPS & Simulation & Anylogic & Medium \\
\hline 2017 & {$[53]$} & MRCPSP-PCF & Forward method & MS Project & High \\
\hline
\end{tabular}

He and Zhang [49] analyzed dynamic priority scheduling in their research. The authors have integrated FBHA into MS Project to optimally solve RLP. The said solving algorithm has been integrated into MS Project via VBA programming tool. The proposed approach was executed through three phases, i.e., (i) initialization phase in MS Project with an initial schedule; (ii) schedule optimization phase with VBA-based FBHA; (iii) and output phase in MS Project used to visualize the optimal solution. It was noted that the main advantage of mentioned algorithm is fast computing speed and simple integration with PMT. The solving algorithm was set in a way that avoids premature 
convergence. While this approach facilitates construction scheduling, further research should address enhancing the model to solve more complex RLPs with resource constraints, RLPs with the possibility of activity splitting and RLPs with more than one type of resource.

In their study, Lim et al. [50] developed concurrent construction scheduling simulation method that integrates concurrency principles, critical path method and simulation for solving TCTP. An application that incorporates the said method was developed and named Concurrent Construction Scheduling Simulation System or $\mathrm{C}^{2} \mathrm{~S}^{2}$. This program was applied to import schedule data from Primavera P6 and manually created spreadsheet-based concurrency attributes. The main goal of this approach was to identify optimal overlaps between project activities and compress overall project duration without assigning additional resources and exceeding the project budget. The main limitation of the model is that only FS precedence relations without lag or lead times were included. In future work, hybridizing of heuristics methods with this approach should be considered to increase run time and improve the results.

Wang et al. [51] have shown an alternative approach to project scheduling. The duration estimation interface module (DEI) that was developed in this study integrated BIM, MS Access database, simulation software Stroboscope, MS Excel and MS Project. The DEI module was developed using the VBA programming language to generate a project schedule. The module requires inputting construction conditions, resource productivity data and quantity of available resources. The simulation then examined various resource allocation strategies and as a result, generated a project along with probability of completion time. Simulations were completed using Stroboscope, and results stored in MS Excel so that they can be imported in MS Project. The authors have created 4D animation of the construction process in Autodesk Navisworks based on BIM and generated schedule. While this approach cannot be considered as an optimization method, simulation runs can create different project schedule alternatives so it can be used as a decision-making tool. The paper exposed that further development should consider the inclusion of an additional optimization module to automate the decision-making process.

Jeong et al. [52] developed a BIM-integrated simulation framework to predict productivity dynamics. The system was established as a three-step process, i.e., (i) development of BIM and data extraction, (ii) construction operation simulation and (iii) obtaining productivity dynamics and production plan. BIM was completed in Autodesk Revit equipped with commands developed in Autodesk Revit API and C\# programming language. Data obtained by API was imported to the Anylogic simulation environment and linked to the proposed production process model. While the Anylogic is primarily simulation software, in this case, it can be considered as a PMT for it was employed to store data of production activity process, resource allocation and critical factors on construction site. The schedule data can also be exported from Anylogic to MS Excel, and therefore to MS Project or any other PMT. Additionally, the optimization approach was not mentioned in this research but the Anylogic website [54] states that their software includes OptQuest Optimization Engine, an optimization tool that can apply metaheuristics, evolutionary algorithms, tabu search and scatter search. Therefore, this could be a simple upgrade of this framework so it can be considered in further research.

Delgoshaei et al. [53] presented a new forward method to solve MRCPSP-PCF. In this study, the authors proposed an optimization model that maximizes the net present value (NPV) of a manufacturing project. The model included the possibility of splitting activities and can provide a modified schedule without overallocated resources. The proposed method was developed as stand-alone software and as MS Project macro. The scheduling problems in this study were solved by MATLAB, but presumably can also be solved using MS Project macro. It was found that the suggested method is applicable and can be used to modify overallocated MRCPSPs by splitting non-critical activities. While this approach brought some significant innovations to the field of project management, there were also some minor limitations in terms of defining activities in different modes with their exact duration, occurrence and cash flows; and considering only positive cash flows. 


\section{Optimization-Based BIM Tools}

BIM increasingly more often takes over the role of PMT since it can provide a wider spectrum of valuable data for construction scheduling in one place than conventional tools. However, from a strictly mathematical viewpoint, BIM is not itself an optimization tool until some optimization algorithm is engaged. A dynamic system integrating optimization methods and BIM is considered an active BIM [55]. Table 5 exposes the progress of optimization-based BIM tools developed particularly for construction scheduling.

Table 5. Research on Optimization-Based BIM Tools.

\begin{tabular}{ccccccc}
\hline Year & Ref. & Scheduling Problem & BIM Tool & Optimization Approach & Project Management Tool & Level of Integration \\
\hline 2014 & {$[56]$} & MOSP & $\begin{array}{c}\text { Autodesk } \\
\text { Revit }\end{array}$ & GA & MS Project & Medium \\
\hline 2015 & {$[57]$} & RCPSP & $\begin{array}{c}\text { Autodesk } \\
\text { Revit }\end{array}$ & PSO and simulation & MS Project & Medium \\
\hline 2015 & {$[58]$} & RAPSP & 4D CAD & Fuzzy theory and GA & 4D CAD & Medium \\
\hline 2018 & {$[59]$} & MRCPSP & OBJ file & BC & MS Excel ${ }^{1}$ & Medium \\
\hline 2020 & {$[12]$} & MRCPSP & $\begin{array}{c}\text { Autodesk } \\
\text { Revit and 4D } \\
\text { BIM client }\end{array}$ & CP $^{2}$ & MS Project and 4D BIM client & Medium \\
\hline
\end{tabular}

Notes: ${ }^{1}$ MS Excel was not primarily designed as a PMT, but it holds capabilities for visualizing, planning and tracking projects; ${ }^{2} \mathrm{CP}$ is executed through the IBM's optimization engine called CP Optimizer [60], which employs several core techniques (a.e. depth first tree search, constraint propagation, large neighborhood search, machine learning, GA, etc.).

In their study, Said and El-Rayes [56] introduced automated multi-objective construction logistics optimization system named AMCLOS. The system was implemented using MS Visual Studio C++ in five main modules, including a module to read input data from the IFC file of project's BIM and a module to import a schedule from MS Project. In the relational database model, BIM geometry attributes must be linked to activities from a schedule. The construction logistics planning module was used to formulate an optimization model and apply GA to solve the optimization problem. The user interface module was developed to facilitate the use of the AMCLOS system and serves as a backbone for data transfer between the rest of the modules. The objectives of this system were minimization of overall logistics cost and minimization of project schedule criticality. The authors said that the further development of the system should aim at solving different construction scheduling problems and establishing a cloud-based collaboration environment.

Liu et al. [57] established an automated system to solve RCPSPs that consists of three main components, i.e., (i) input module in MS Access with resource information and work breakdown structure (WBS); (ii) building 3D design created in Autodesk Revit; and (iii) schedule generated in MS Project. The components were connected through the Autodesk Revit API that was programmed in C\#. Autodesk Revit included add-on for structural and spatial relationship analysis, a process simulation model run in Symphony and PSO model to generate optimal construction schedule. The proposed approach was validated through scheduling for panelized construction and generated an expected schedule that reduces human error in the project management process. Limitation of the said approach was that the duration and productivity of work packages were estimated, the weather and construction site conditions were not considered, and part of the simulation network still had to be manually formed.

Moon et al. [58] developed a 4D CAD system that uses fuzzy theory and GA to solve specific RCPSP type called RAPSP. This study presented the schedule overlapping ratio (SOR) as a backbone of the proposed method that determines periods of simultaneous task execution using information from the database. The system contained all project input parameters in the MS Access database file. BIM projects and schedule files could be imported and saved in a database. The system was accommodated with additional modules for initial schedule generation, an algorithm for finding activity overlaps, risk analysis that uses fuzzy theory and GA optimization module to minimize the number of overlap activities. After the optimal schedule is obtained, a module for $4 \mathrm{D}$ simulation can 
be used to visualize the construction process with an option of marking the activities risk by color. The 4D CAD system was verified on an example of tunnel construction for the railway project where it was applied to solve frequent schedule overlaps. However, by minimizing SOR, some activities can be shifted through schedule, though there is no concern about utilized resources or activities that are more effective if scheduled in parallel. Moreover, additional costs and overall project duration increase were not examined.

The study by Volk et al. [59] presented a new approach to deconstruction project planning. The authors developed a system called ResourceApp that can recreate a 3D model of a building. The ResourceApp system was demonstrated on the example of a hospital in Germany. First, the building was recorded with Microsoft Kinect and graphical building reconstruction was obtained with automatically recognized building elements. Then, the system required user input on building parameters and general building information so that building inventory can be obtained, i.e., concrete mass, building equipment, etc. Additionally, deconstruction activities were generated. Finally, the optimization model was developed in MATLAB to minimize deconstruction's overall duration under resource constraints. The MRCPSP was modeled as a MILP in MATLAB and solved applying the BC algorithm within CPLEX solver. The optimization output was exported to MS Excel and presented as a Gantt schedule. The authors point out the limitations of this approach in terms of mobility of the motion sensor and its accuracy. Further research should consider the implementation of a wearable helmet equipped with 3D scanning devices. A study by Pučko et al. [61] suggests similar technology for monitoring construction progress comparing BIM as-built and BIM as-designed. This could be a further step towards automating the construction process. The project manager could go through the construction site with such a helmet, create a point cloud and compare the data with BIM. Then the given results could be used for updating the optimization model and creating an optimal schedule at any stage of construction process.

Wang et al. [12] proposed an integrated approach that facilitates data flow from BIM to the MRCPSP model for construction scheduling. The authors developed 4D-BIM software that can import and export various file formats, i.e., MS Project files, MS Excel files, IFC, OBJ, DXF, 3DXML; and support model management with $3 \mathrm{D} / 4 \mathrm{D}$ visualizations. The capabilities were demonstrated on the example of a 22-story residential building in China. BIM was completed in Autodesk Revit, exported to IFC format and imported to 4D-BIM application. The software can recognize BIM elements by element codes and determine object-oriented activities that should be included in the scheduling problem. Moreover, the duration of activities was not set as fixed; the model uses a work package templates database that allows utilizing various resource combinations to obtain different task durations. Finally, the optimization model was designed with IBM ILOG CPLEX Optimization Studio equipped with the NET API for the proposed mathematical model. The optimal schedule can then be exported to MS Project to validate the feasibility and efficiency of the schedule or to create $4 \mathrm{D}$ visualization of the construction process. The authors consider extending the proposed information model to the cost estimation system and safety management tool. Additionally, the heuristics methods and automatic progress tracking technology were proposed.

\section{Conclusions}

This paper presented the achievements survey on the integration of optimization and PMT that allow sustainable construction scheduling, particularly in terms of continuous optimal time and resource allocation throughout the project life cycle. Following a brief introduction, the optimization platform for construction scheduling was given in the article. Focusing on construction scheduling, an in-depth achievements survey on the integration of heuristics methods, mathematical programming and special solving methods with conventional PMT as well as optimization-based BIM tools was then performed, and findings were reported. The survey led to the following conclusions and suggestions for further research. 
It was revealed very early on that the integration of heuristics methods and PMT attracted a considerable interest among researchers. Some studies have attained a high level of automation in the process of integration. With a few exceptions, MS Project was mainly used as PMT, mostly because of widespread use among project managers, so that proposed optimization techniques can be easier applied in practice. The most common integration approaches can be highlighted here, i.e., (i) integration of heuristic algorithm via VBA in MS Project, (ii) model developed in MS Excel and GA applied through Solver add-in, (iii) development of a new environment that contains heuristic algorithm and has the ability to read and store data to PMT, and (iv) development of data transfer system that allows employing stand-alone optimization tools based on data stored in PMT. The highest level of automation was mainly obtained applying the first approach which required no data transfer, so the optimal results can be generated within PMT. When it comes to the optimization approach, attention was often directed to GAs since they can solve a large share of scheduling problems in a reasonable time. Heuristics methods have been applied to the most common project scheduling problems, from TCTPs, RLPs and RAPs to RCPSPs and multi-objective analysis. Future studies should discuss the automation of the process of generating the initial project schedule based on the construction design. Therefore, BIM tools that facilitate data collection should be considered.

On the other hand, slightly less attention has been paid to the integration of mathematical programming methods and PMT. Mathematical programming is stricter to what it can represent than other techniques and provides the exact optimal solution to the scheduling problem as it has been modeled. Some of the most often used integration techniques can be put in the fore here: (i) optimization model developed in MS Excel, solved by Simplex LP solver and data modified for PMT, (ii) extension of the PMT capabilities with external programming to accommodate exact algorithms, and (iii) use of algebraic modeling languages to develop the optimization model that can be integrated with PMT. Optimization models exposed in Section 4 were mainly established with LP and MILP formulations while the employed solving methods included simplex, BB and BC algorithms. Researchers were mainly focused on RCPSPs and TCTPs, so there exists a possibility for further research on RLPs, RAPs and MRCPSPs among others. Besides, the exact multi-objective optimization of construction schedules is also quite an open area to be investigated. This section leaves plenty of space for further research in the field of integrating the PMT with commercial algebraic modeling languages. The main challenges with this type of integration is how to take the data from PMT to the modeling language and how to develop the optimization model so that it would be generally applicable. Construction schedules are dynamic and often require to be updated with many different changes as they are in use. In this way, it would be desirable for the optimization model to be able to incorporate all that changes without excessive manual programming.

Section 5 brought the implementation of dynamic priority-driven scheduling and the simulation algorithms to the PMT. The studies with dynamic priority rules had an approach of integrating solving algorithm into the MS Project through the VBA-based programming, so that the solution process was highly automated. On the other hand, the simulation models required the use of specialized tools for this purpose, and the data flow between simulation model and PMT was found not to be fully automated. Although simulation is not an optimization method, a probabilistic model can be developed to support the decision-making process by observing the relationship between input and output over many realizations. Simulation-based solution approaches are yet to be explored in construction scheduling, but simulation complexity could become a bottleneck for applicability. The examples of BIM involvement in process of collecting input data have been demonstrated, though there is a lack of automation in terms of output to generally used PMT. Special solving methods mentioned above should be employed in future studies on multi-objective scheduling problems.

Introduction of BIM tools facilitated the process of creating optimization models for construction scheduling. All of the parameters that would need to be calculated manually became available instantly through utilization of BIM tools. The well-developed models already contain information about material and spatial properties of separate objects. The resources can be allocated to the objects within 
BIM tool; activities and their durations can be generated so the initial plan of construction can also be created in BIM. An issue addressed in studies was how to extract all required parameters for an optimization model from BIM. One of the suggested approaches was to embed the optimization model trough API function and perform optimization within BIM tool and export data to PMT for further project management. Another approach was to develop user interface that can read and store the data about the model and the schedule. After the data is entered into the system, the optimization can be performed to create output to PMT or to create visualization of construction process. BIM from the studies was mostly completed in Autodesk Revit or other BIM tools that can store the model in standardized IFC format. An opportunity for further research, in context of schedule updating, is use of point cloud created with 3D scanner technology to establish BIM of construction as-build. Scheduling models were mainly developed for RCPSPs or some of the derivatives such as MRCPSPs and RAPSPs, so there exists an open field for investigating RAPs, RLPs, TCTPs and extension to multi-objective analysis.

General applicability of integrations between optimization and PMT as they were identified in present survey seems to be brought down with data compliance between software used. The open-ended challenges found are high complexity of such optimization models and the fact that their establishment requires certain programming skills. Aware that optimal construction scheduling is about solving various combinatorial problems, the generation of the superstructure of solution alternatives (a.e. time-cost-resource options related to different execution modes for activities as well as variants for the production process implementation) still requires to be done attentively, in order to take full advantages of the optimization model.

To accelerate the convergence of the optimal scheduling solution, it is mostly advisable to launch the optimization algorithm from a feasible point, if possible. When the capacity of a personal computer or workstation is found to be insufficient, the accelerated solution convergence can be achieved by running the optimization model on a more powerful machine available online, for example, a public supercomputer. This may prove particularly suitable for scheduling complex, high-value construction projects where even a single application of such a model could create a benefit. Nevertheless, the undoubted potential of integrated optimization models to assure not only optimized schedules but also sustainable scheduling process throughout the construction project life cycle encourages intensifying research on their further development.

Author Contributions: Conceptualization, B.D. and U.K.; methodology, B.D. and U.K.; validation, M.G. and U.K.; formal analysis, B.D.; investigation, B.D.; writing — original draft preparation, B.D. and U.K.; writing-review and editing, M.G. and U.K.; supervision, M.G. and U.K.; project administration, U.K.; funding acquisition, M.G. and U.K. All authors have read and agreed to the published version of the manuscript.

Funding: The APC was funded by discount vouchers.

Acknowledgments: The authors acknowledge the financial support from the Slovenian Research Agency (Research Core Funding No. P2-0129). This publication is a part of research project “Monitoring of Tower Cranes' Key Reliability Parameters for Safety and Performance-MonitorCRANE" funded by the Faculty of Civil Engineering and Architecture Osijek.

Conflicts of Interest: The authors declare no conflict of interest.

\section{References}

1. U.S. Environmental Protection Agency. Green Building. Available online: https://archive.epa.gov/ greenbuilding/web/html/about.html (accessed on 14 April 2020).

2. Baruah, S.; Burns, A. Sustainable scheduling analysis. In Proceedings of the 27th IEEE International Real-Time Systems Symposium (RTSS'06), Rio de Janeiro, Brazil, 5-8 December 2006; pp. 159-168.

3. Hermarij, J. Better Practices of Project Management Based on IPMA Competences, 4th ed.; Van Haren Publishing: Hertogenbosch, The Netherlands, 2016.

4. Srdić, A.; Šelih, J. Delays in construction projects: Causes and mitigation. Organ. Technol. Manag. Constr. Int. J. 2015, 7, 1383-1389. [CrossRef] 
5. Aslam, M.; Baffoe-Twum, E.; Saleem, F. Design changes in construction projects-Causes and impact on the cost. Civ. Eng. J. 2019, 5, 1647-1655. [CrossRef]

6. Hartmann, S.; Briskorn, D. A survey of variants and extensions of the resource-constrained project scheduling problem. Eur. J. Oper. Res. 2010, 207, 1-14. [CrossRef]

7. Habibi, F.; Barzinpour, F.; Sadjadi, S.J. Resource-constrained project scheduling problem: Review of past and recent developments. J. Proj. Manag. 2018, 3, 55-88. [CrossRef]

8. Ballesteros-Pérez, P.; Elamrousy, K.M.; González-Cruz, M.C. Non-linear time-cost trade-off models of activity crashing: Application to construction scheduling and project compression with fast-tracking. Autom. Constr. 2019, 97, 229-240. [CrossRef]

9. Servranckx, T.; Vanhoucke, M. A tabu search procedure for the resource-constrained project scheduling problem with alternative subgraphs. Eur. J. Oper. Res. 2019, 273, 841-860. [CrossRef]

10. El-Sayegh, S. Resource levelling optimization model considering float loss impact. Eng. Constr. Architect. Manag. 2018, 25, 639-653. [CrossRef]

11. Tran, D.H.; Long, L.D. Project scheduling with time, cost and risk trade-off using adaptive multiple objective differential evolution. Eng. Constr. Architect. Manag. 2018, 25, 623-638. [CrossRef]

12. Wang, H.W.; Lin, J.R.; Zhang, J.P. Work package-based information modeling for resource-constrained scheduling of construction projects. Autom. Constr. 2020, 109, 1-20. [CrossRef]

13. Klanšek, U. Mixed-integer nonlinear programming model for nonlinear discrete optimization of project schedules under restricted costs. J. Constr. Eng. Manag. 2016, 142, 04015088. [CrossRef]

14. Cajzek, R.; Klanšek, U. Cost optimization of project schedules under constrained resources and alternative production processes by mixed-integer nonlinear programming. Eng. Constr. Architect. Manag. 2019, 26, 2474-2508. [CrossRef]

15. Venkrbec, V.; Galić, M.; Klanšek, U. Construction process optimisation-Review of methods, tools and applications. Građevinar 2018, 70, 593-606. [CrossRef]

16. Obradović, D. Review of nature-inspired optimization algorithms applied in civil engineering. E-GFOS 2018, 9, 74-88. [CrossRef]

17. Hegazy, T. Optimization of construction time-Cost trade-off analysis using genetic algorithms. Can. J. Civ. Eng. 1999, 26, 685-697. [CrossRef]

18. Hegazy, T. Optimization of resource allocation and leveling using genetic algorithms. J. Constr. Eng. Manag. 1999, 125, 167-175. [CrossRef]

19. Leu, S.-S.; Yang, C.-H.; Huang, J.-C. Resource leveling in construction by genetic algorithm-based optimization and its decision support system application. Autom. Constr. 2000, 10, 27-41. [CrossRef]

20. Crawford, J.; Do, J.; Leduc, C.; Malik, A.; Gormley, K.; Luebke, E.; Scherer, W. SWORD: The latest weapon in the project selection arsenal. In Proceedings of the 2003 IEEE Systems and Information Engineering Design Symposium, Charlottesville, VA, USA, 24-25 April 2003; pp. 95-100.

21. Sriprasert, E.; Dawood, N. Genetic algorithms for multi-constraint scheduling: An application for the construction industry multi-constraint scheduling. In Proceedings of the CIB w78 Conference 2003, Auckland, New Zealand, 23-25 April 2003; p. 12.

22. Hegazy, T.; Kassab, M. Resource optimization using combined simulation and genetic algorithms. J. Constr. Eng. Manag. 2003, 129, 698-705. [CrossRef]

23. Zheng, D.X.M.; Ng, S.T.; Kumaraswamy, M.M. Applying pareto ranking and niche formation to genetic algorithm-based multiobjective time-cost optimization. J. Constr. Eng. Manag. 2005, 131, 81-91. [CrossRef]

24. Dawood, N.; Sriprasert, E. Construction scheduling using multi-constraint and genetic algorithms approach. Constr. Manag. Econ. 2006, 24, 19-30. [CrossRef]

25. Kandil, A.; El-Rayes, K. MACROS: Multiobjective automated construction resource optimization system. J. Manag. Eng. 2006, 22, 126-134. [CrossRef]

26. Zhang, Y.; Ng, S.T.; Kumaraswamy, M.M. Applying ant colony system to solve construction time-cost trade-off problem. In Proceedings of the CME 2007 Conference-Construction Management and Economics: 'Past, Present and Future', Berkshire, UK, 16-18 July 2007; pp. 1227-1233.

27. Lancaster, J.; Cheng, K. Optimization of the hydrotesting sequence in tank farm construction using an adaptive genetic algorithm with stochastic preferential logic. Proc. Instit. Mech. Eng. Part B J. Eng. Manuf. 2008, 222, 367-371. [CrossRef] 
28. Lu, M.; Lam, H.-C.; Dai, F. Resource-constrained critical path analysis based on discrete event simulation and particle swarm optimization. Autom. Constr. 2008, 17, 670-681. [CrossRef]

29. Zülch, G.; Steininger, P.; Gamber, T.; Leupold, M. Generating, benchmarking and simulating production schedules-From formalisation to real problems. In Proceedings of the -Winter Simulation Conference, Austin, TX, USA, 13-16 December 2009; pp. 2238-2249.

30. Kim, J.-L.; Ellis, R.D., Jr. A framework for integrating elitist GA and project management tool. In Proceedings of Building a Sustainable Future-Proceedings of the 2009 Construction Research Congress, Seattle, DC, USA, 5-7 April 2009; pp. 1096-1104.

31. Anagnostopoulos, K.P.; Koulinas, G.K. A simulated annealing hyperheuristic for construction resource levelling. Constr. Manag. Econ. 2010, 28, 163-175. [CrossRef]

32. Anagnostopoulos, K.P.; Koulinas, G.K. A genetic hyperheuristic algorithm for the resource constrained project scheduling problem. In Proceedings of the 2010 IEEE World Congress on Computational Intelligence, WCCI 2010-2010 IEEE Congress on Evolutionary Computation, Barcelona, Spain, 18-23 July 2010; pp. 1-6. [CrossRef]

33. Koulinas, G.K.; Anagnostopoulos, K.P. Construction resource allocation and leveling using a threshold accepting-based hyperheuristic algorithm. J. Constr. Eng. Manag. 2012, 138, 854-863. [CrossRef]

34. Zhang, Y.; Ng, S.T. An ant colony system based decision support system for construction time-cost optimization. J. Civ. Eng. Manag. 2012, 18, 580-589. [CrossRef]

35. Anagnostopoulos, K.; Koulinas, G. Resource-constrained critical path scheduling by a GRASP-based hyperheuristic. J. Comput. Civ. Eng. 2012, 26, 204-213. [CrossRef]

36. Guedes, A.; Tereso, A. Scheduling with MS Project: An add-in. In Proceedings of the 6th Multidisciplinary International Conference on Scheduling: Theory and Applications (MISTA 2013), Gent, Belgium, 27-30 August 2013; pp. 707-710.

37. Moselhi, O.; Roofigari-Esfahan, N. Project schedule compression: A multi-objective methodology. Constr. Innov. 2013, 13, 374-393. [CrossRef]

38. Gonzalez-Sieira, A.; Bugarin, A.; Mucientes, M.; Moran, J. A tabu search optimization module for scheduling: Design and integration in the open source tool LibrePlan for project management. In Proceedings of the Iberian Conference on Information Systems and Technologies, Lisboa, Portugal, 19-22 June 2013.

39. Koulinas, G.K.; Anagnostopoulos, K.P. A new tabu search-based hyper-heuristic algorithm for solving construction leveling problems with limited resource availabilities. Autom. Constr. 2013, 31, 169-175. [CrossRef]

40. Karova, M.; Penev, I.; Todorova, G.; Todorova, M. Genetic algorithm for managing project activities system. In Proceedings of the 2014 International Conference on Mathematics and Computers in Sciences and in Industry, Varna, Bulgaria, 13-15 September 2014; pp. 267-271.

41. Tereso, A.; Guedes, A.; Cascais, A. A computer application for scheduling in MS Project. Comput. Sci. Appl. 2014, 1, 309-318.

42. Karova, M.; Todorova, G.; Penev, I.; Todorova, M. Managing project activities system using genetic algorithm. In Proceedings of the Recent Advances in Civil Engineering and Mechanics, Florence, Italy, 22-24 November 2014; pp. 218-224.

43. Kaiafa, S.; Chassiakos, A.P. A genetic algorithm for optimal resource-driven project scheduling. Procedia Eng. 2015, 123, 260-267. [CrossRef]

44. Eid, M.S.; Elbeltagi, E.E.; El-Adaway, I.H. Simultaneous multi-criteria optimization for scheduling linear infrastructure projects. Int. J. Constr. Manag. 2018, 1-15. [CrossRef]

45. Hebert, J.E.; Deckro, R.F. Combining contemporary and traditional project management tools to resolve a project scheduling problem. Comput. Oper. Res. 2011, 38, 21-32. [CrossRef]

46. Tiwari, S.; Johari, S. Project scheduling by integration of time cost trade-off and constrained resource scheduling. J. Inst. Eng. India Ser. A 2015, 96, 37-46. [CrossRef]

47. Siganporia, C.C. Strategic Biopharmaceutical Production Planning for Batch and Perfusion Processes; University College London: London, UK, 2016.

48. Valenko, T.; Klanšek, U. An integration of spreadsheet and project management software for cost optimal time scheduling in construction. Organ. Technol. Manag. Constr. Int. J. 2017, 9, 1627-1637. [CrossRef]

49. He, L.; Zhang, L. Dynamic priority rule-based forward-backward heuristic algorithm for resource levelling problem in construction project. J. Oper. Res. Soc. 2013, 64, 1106-1117. [CrossRef] 
50. Lim, T.K.; Yi, C.Y.; Lee, D.E.; Arditi, D. Concurrent construction scheduling simulation algorithm. Computer-Aided Civ. Infrastruct. Eng. 2014, 29, 449-463. [CrossRef]

51. Wang, W.C.; Weng, S.W.; Wang, S.H.; Chen, C.Y. Integrating building information models with construction process simulations for project scheduling support. Autom. Constr. 2014, 37, 68-80. [CrossRef]

52. Jeong, W.; Chang, S.; Son, J.; Yi, J.-S. BIM-integrated construction operation simulation for just-in-time production management. Sustainability 2016, 8, 1106. [CrossRef]

53. Delgoshaei, A.; Rabczuk, T.; Ali, A.; Ariffin, M.K.A. An applicable method for modifying over-allocated multi-mode resource constraint schedules in the presence of preemptive resources. Ann. Oper. Res. 2017, 259, 85-117. [CrossRef]

54. AnyLogic. Simulation Modeling Software Tools \& Solutions for Business. Available online: https://www. anylogic.com/ (accessed on 10 March 2020).

55. Dasović, B.; Galić, M.; Klanšek, U. Active BIM approach to optimize work facilities and tower crane locations on construction sites with repetitive operations. Buildings 2019, 9, 21. [CrossRef]

56. Said, H.; El-Rayes, K. Automated multi-objective construction logistics optimization system. Autom. Constr. 2014, 43, 110-122. [CrossRef]

57. Liu, H.; Al-Hussein, M.; Lu, M. BIM-based integrated approach for detailed construction scheduling under resource constraints. Autom. Constr. 2015, 53, 29-43. [CrossRef]

58. Moon, H.; Kim, H.; Kamat, V.R.; Kang, L. BIM-based construction scheduling method using optimization theory for reducing activity overlaps. J. Comput. Civ. Eng. 2015, 29, 1-16. [CrossRef]

59. Volk, R.; Luu, T.H.; Mueller-Roemer, J.S.; Sevilmis, N.; Schultmann, F. Deconstruction project planning of existing buildings based on automated acquisition and reconstruction of building information. Autom. Constr. 2018, 91, 226-245. [CrossRef]

60. IBM Corporation. IBM ILOG CPLEX Optimization Studio CP Optimizer User's Manual. Available online: https://www.ibm.com/support/knowledgecenter/SSSA5P_12.8.0/ilog.odms.studio.help/pdf/ usrcpoptimizer.pdf (accessed on 23 March 2020).

61. Pučko, Z.; Šuman, N.; Rebolj, D. Automated continuous construction progress monitoring using multiple workplace real time 3D scans. Adv. Eng. Inf. 2018, 38, 27-40. [CrossRef]

(C) 2020 by the authors. Licensee MDPI, Basel, Switzerland. This article is an open access article distributed under the terms and conditions of the Creative Commons Attribution (CC BY) license (http://creativecommons.org/licenses/by/4.0/). 